\title{
The Application of Combination Forecast Model for Subgrade Settlement Forecasting in the High-speed Railway
}

\author{
Gao Hui ${ }^{1}$, Huang Jun ${ }^{2}$, Zhang Rui ${ }^{3, *}$ and Zhu run-xia ${ }^{3}$ \\ ${ }^{1}$ School of Electrical and Information Engineering, Heilongjiang Institute of \\ Technology, Harbin, Heilongjiang, China 150050 \\ ${ }^{2}$ Faculty of Geosciences and Environmental Engineering, Southwest Jiaotong \\ University, Chengdu, Sichuan, China 610031 \\ ${ }^{3}$ School of Automation, Harbin University of Science and Technology, Harbin, \\ Heilongjiang, China 150080 \\ zr_gh@sina.com
}

\begin{abstract}
High-speed railway subgrade settlement forecasting is an important part to ensure ride comfort and stability of the high-speed railway line. Based on the measured settlement data of Lan-Xin Railway DK649+500D-K650+000 section, three kinds of single forecast methods, including Hyperbolic method, Three-point method and Asaoka method are adopted for the high-speed railway subgrade settlement forecasting. The least squares support vector machine in adopted for its unique advantages in solving small sample data and nonlinear data. Combination forecast model is established by setting different weights of the single forecast methods. Simulation results show that the systematic errors in the Combination forecast model are less than that in the single forecast methods. Compared with the single forecast methods, the Combination forecast model has higher forecasting accuracy and better applicability.
\end{abstract}

Keywords: Subgrade settlement forecasting; Single forecast model; Least squares support vector machine; Combination forecast model

\section{Introduction}

Safe and stable operation of high-speed rail requires non-ballasted track subgrade kept uniform horizontal plane in continuous, the appropriate design measures have been taken in the design to solve the embankment settlement problems, a long-term, no regularity, complexity, and other characteristics. However, affected by many factors, the current settlement calculation accuracy is not enough to satisfy the demand of controlling the settlement after laying non-ballasted track. Settlement forecasting methods are mainly divided into two categories: curve fitting and artificial intelligence. Hyperbolic, Exponential curve, Three-point method, Asaoka method and the Poisson curve belong to curve fitting $[1,2]$. Hyperbolic and exponential curve only reflect the general rules of subgrade settlement, there isn't reflect the complex characteristics of subgrade settlement, Three-point method has better adaptability of the cases of data fluctuation, Asaoka method less affected by volatility of the original data, and the selection of time interval and the starting point almost no effect on the related value; Artificial neural network is a method of artificial intelligence[3], which improves the accuracy and reliability of the railway embankment settlement forecasting in some extent, but the drawback of this method is the convergence rate is low, easy to fall into 
local minima. Based on the criterion of structural risk minimization, LS-SVM improves the forecasting ability of the learning machine, can solve the practical problems of small samples, nonlinearity, high dimension and local minima $[4,5]$.

This paper takes full advantage of the Hyperbolic method, Three-point method and Asaoka's fitting characteristics established single forecast models of subgrade settlement. Then using LS-SVM to assign different rights to the single forecast methods to establish the Combined Forecasting model[6], which is used for subgrade settlement forecasting of subgrade DK649+500D-K650+000 section of Lan-Xin Railway, while the experimental results shows that this Combination Forecast model has better forecasting accuracy than single forecast model.

\section{Single Forecasting Model Construction}

\subsection{The Hyperbolic Method}

Theoretical basis of Hyperbolic method is that the process of subgrade changing as the rate of hyperbola trend. The process begin after the loading finished, and the settlement $S$ to $t$ ca can be expressed as:

$$
\begin{gathered}
S_{t}=S_{0}+\frac{t-t_{0}}{a+b\left(t-t_{0}\right)} \\
S_{\infty}=S_{0}+\frac{1}{b}
\end{gathered}
$$

where $S_{\infty}$ represents the final settlement value; $S_{0}$ is the initial monitoring value of the load completed; $\alpha, b$ is the regression parameter.

\subsection{Three-point Method}

Three-point method, also known as the degree of consolidation ratio logarithmic method, expression is as follows:

$$
S_{t}=S_{d} \alpha e^{-\beta t}+S_{\infty}\left(1-\alpha e^{-\beta t}\right)
$$

Three time points $\left(t_{1}, S_{1}\right),\left(t_{2}, S_{2}\right),\left(t_{3}, S_{3}\right)$ are taken from the samples settlement curve, and fulfill $t_{3}-t_{2}=t_{2}-t_{1}$. The three time point values take into the above equation can obtain the values of $S_{\infty}, S_{d}, \beta$, but the instantaneous settlement value $S_{d}$ contains the unknown parameter $\alpha$, based on the theory and many experiments the value select $8 / \pi^{2}$.

The three point data selection should be selected after the settlement curve inflection point, should avoid select the observation point with continuous or jumping larger magnitude on the settlement curve, should reflect the overall trend of settlement curve.

\subsection{Asaoka Method}

Asaoka theory is that the volume strain consolidation equation of Mikasa can be expressed as:

$$
S+a_{1} \frac{d s}{d t}+a_{2} \frac{d^{2} s}{d t^{2}}+\ldots+a_{n} \frac{d^{n} s}{d t^{n}}=b
$$

where $\mathrm{S}$ is the total amount of consolidation settlement; $a_{1}, a_{2}, \ldots a_{n}$ represent constant coefficient of consolidation and soil boundary conditions. Asaoka method basic idea is to use existing observations to solve these coefficients, and then use these parameters to build forecast models to estimate the final settlement value. 
According to the length and the corresponding settlement known sample is divided into $t_{j}=j \Delta t(j=1,2 \ldots l)$, and $\Delta t$ is constant, $S_{j}=S\left(t_{j}\right)$. In practical engineering calculations often take an order, so the subgrade settlement can be expressed as:

$$
\begin{aligned}
& S+a_{1} \frac{d s}{d t}=b \\
& S_{j}=\beta_{0}+\beta_{1} S_{j-1}
\end{aligned}
$$

where $\mathrm{S}$ is an unknown quantity, $\beta_{0}$ is the settling volume, $\beta_{1}$ is one dimension constant, $S_{0}$ and $S_{\infty}$ represent the initial foundation settlement and final settlement, then the general solution for the above formula is:

$$
S(t)=S_{\infty}-\left(S_{\infty}-S_{0}\right) e^{-a_{1} t}
$$

Order $\lim _{t_{j} \rightarrow \infty} t=t_{j}$ and $S_{j}=S_{j-1}$, then the final settlement is: $S_{\infty}=\frac{\beta_{0}}{1-\beta_{1}}$

\section{Combined Forecasting Model}

\subsection{LS-SVM}

The basic idea of support vector machines (SVM) is through a nonlinear mapping the input space is mapped into a high dimensional feature space, where a linear regression is performed. This approach avoids the tedious dot product operations. Least squares support vector machine (LS-SVM) is an improvement of SVM [7], it can transform the inequality constraint rules into equality constraints and transform the objective optimization problem into the quadratic programming problem.

$$
\min _{w, b, \varepsilon} J(w, \varepsilon)=\frac{1}{2}\|w\|^{2}+\frac{C}{2} \sum_{i=1}^{n} e_{i}^{2}
$$

The constraint condition is:

$$
y_{i}=w^{T} \varphi\left(x_{i}\right)+b+e_{i}, i=1,2, \ldots n
$$

where $e_{i} \in R$ is error variable, $C>0$ is penalty coefficient balance of confidence and loss of function.

Using duality theory, LS-SVM can boils down to solving a system of linear equations:

$$
\left[\begin{array}{cc}
K+C^{-1} I & e l \\
e l^{T} & 0
\end{array}\right]\left\{\begin{array}{l}
a \\
b
\end{array}\right\}=\left\{\begin{array}{l}
y \\
0
\end{array}\right\}
$$

where $y=\left(y_{1}, y_{2}, \ldots y_{n}\right)^{T}, a=\left(a_{1}, a_{2}, \ldots a_{n}\right)^{T}, e l=(1,1, \ldots, n)^{T}, \mathrm{I}$ is an unit matrix of order $\mathrm{n} ; \mathrm{K}$ is the kernel function matrix, using a radial basis function, whose elements are:

$$
\mathrm{k}_{\mathrm{ij}}=k\left(x_{i}, x_{j}\right)=\exp \left(-\frac{\left\|x-x_{i}\right\|^{2}}{\sigma^{2}}\right), i, j=1,2, \ldots, n
$$

By equation (10), $a$ and $\mathrm{b}$ can be worked out, and the fitting function of LS-SVM is:

$$
y(x)=\sum_{i=1}^{n} a_{i} k\left(x, x_{i}\right)+b
$$




\subsection{Combination Forecast Model Construction}

The Combination Forecast model consists of LS-SVM and three single forecast models of Hyperbolic method, Three-point method and Asaoka method, the basic idea is: first, for each point, calculating the predicted value of each single forecast models, and treating it as various components of the input vector of LS-SVM, while the observed value of the corresponding point as the output vector of LS-SVM. Through training and optimizing LS-SVM, the SVM of Combination Forecast model is established. Calculating the predicted value of the corresponding point of each single forecast model, and using the SVM of Combination Forecast mode created before, output the final predicted value. The construction of Combination Forecast model is shown as Figure 1.

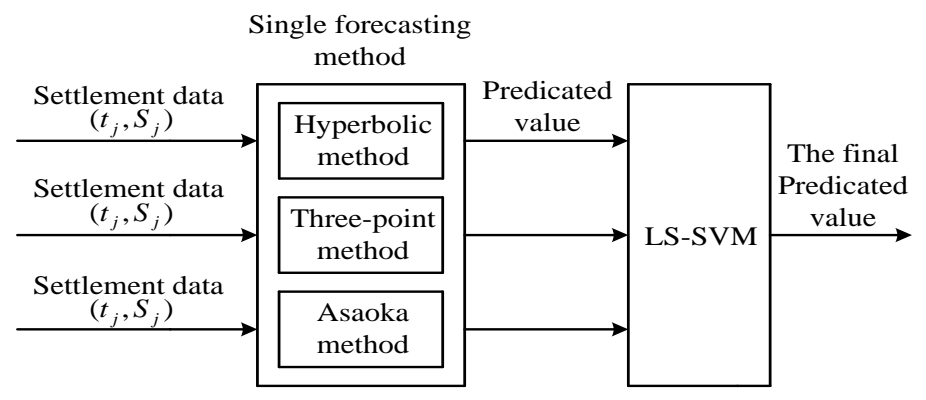

Figure 1. The Construction of Combination Forecast Model

\section{The Application of Combined Forecasting Model}

In this paper, using observed settlement data of the section DK649+500D- K650+000 of Lan-Xin Railway to build the Combination Forecast model. 17 sets of data for the model build, four sets of data used to verify the forecasting effects, settlement data shown in Table 1.

Table 1. Settlement Data

\begin{tabular}{c|c|c|c|c|c}
\hline No. & $\begin{array}{c}\text { Observation } \\
\text { Data }\end{array}$ & $\begin{array}{c}\text { Settlement } \\
\text { Value }(\mathrm{mm})\end{array}$ & No. & $\begin{array}{c}\text { Observation } \\
\text { Data }\end{array}$ & $\begin{array}{c}\text { Settlement } \\
\text { Value }(\mathrm{mm})\end{array}$ \\
\hline 1 & 30 & 0.57 & 12 & 720 & 11.94 \\
\hline 2 & 60 & 2.45 & 13 & 780 & 13.22 \\
\hline 3 & 120 & 4.30 & 14 & 840 & 13.20 \\
\hline 4 & 180 & 4.62 & 15 & 900 & 13.46 \\
\hline 5 & 240 & 6.36 & 16 & 960 & 14.00 \\
\hline 6 & 300 & 7.32 & 17 & 1020 & 14.25 \\
\hline 7 & 360 & 8.66 & \multicolumn{3}{|c}{ The Forecast Data } \\
\hline 8 & 420 & 8.85 & 18 & 1080 & 14.40 \\
\hline 9 & 480 & 10.00 & 19 & 1140 & 14.25 \\
\hline 10 & 540 & 11.55 & 20 & 1200 & 14.53 \\
\hline 11 & 600 & 11.49 & 21 & 1260 & 14.71 \\
\hline
\end{tabular}

\subsection{Single Forecast Model Validation}

4.1.1 Build forecast model of Hyperbolic: Taking the initial time $t_{0}=0$, the settlement value $S_{0}=0$. According to the equation 1 and 2 , the fitting parameter values $a=-0.6343$, $\mathrm{b}=0.0263$. The Hyperbolic model can be expressed as: 


$$
\mathrm{S}_{\mathrm{t}}=\frac{t}{-0.6343+0.0263 t}
$$

4.1.2 Build forecast model of Three-point: Selection of three sets values of equal time intervals: $(240,6.36) 、(600,11.49)$ and $(960,14.00)$, according to the equation 3 , working out $\beta=0.0020 ; \mathrm{S}_{\infty}=16.400 ; S_{d}=-2.1320$, The formula for the final forecast can be expressed as:

$$
\mathrm{S}_{\mathrm{t}}=-2.1320 \times \frac{8}{\pi^{2}} \times e^{-0.0020 t}+16.40 \times\left(1-\frac{8}{\pi^{2}} \times e^{-0.0020 t}\right)
$$

4.1.3 Build forecast model of Asaoka: In the process of modeling, selection of the time interval directly affects the accuracy of the forecast method, after many searching experiments, proved the best fitting is $\Delta t=35$. According to the selection of settlement value and the principles of solving, the Asaoka model can be expressed as:

$$
S(t)=14.7826 \times\left(1-e^{-0.0027 t}\right)
$$

\subsection{Accuracy Evaluation of Combination Forecast Model}

According to error theory[8], evaluation indexes of evaluating the accuracy of the forecast model, including the root mean square error (RMSE), correlation coefficient (CC), mean absolute percentage error (MAPE) and so on. We define RMSE as follows

$$
R M S E=\sqrt{\frac{\sum_{i=1}^{n}\left(y_{t}-y\right)^{2}}{n}}
$$

it may reflects the deviation between the forecasted and measured values. Correlation coefficient can be formulated as

$$
C C=\frac{\sum y y_{t}-\frac{\sum y \sum y_{t}}{n}}{\sqrt{\left(\sum y^{2}-\frac{\left(\sum y\right)^{2}}{n}\right)\left(\sum y_{t}{ }^{2}-\frac{\left(\sum y_{t}\right)^{2}}{n}\right)}}
$$

its result may reflect the degree of correlation between forecasted and measured values. Mean absolute percentage error is described

$$
M A P E=\frac{\sum_{i=1}^{n}\left|\frac{y_{i t}-y_{i}}{y_{i t}}\right|}{n} \times 100 \%
$$

it may reflect the accuracy of forecast results.

\subsection{Verify Combination Forecast Model}

Taking advantage of the Hyperbolic method, Three-point method and Asaoka's fitting characteristics establish single forecast model of subgrade settlement. LS-SVM assigns different rights to the single forecast method to establish the Combination Forecast model. The results of three single forecast methods and Combination Forecast 
model are shown in Table 2, the predictive effect and the relative error of four kinds of forecasting methods are shown in Figure 2 and Figure 3.

Table 2. Four Kinds of Forecast Methods Comparison of Forecast Results

\begin{tabular}{c|c|c|c|c|c|c|c|c|c}
\hline NO. & $\begin{array}{c}\text { Measured } \\
\text { values } \\
(\mathrm{mm})\end{array}$ & \multicolumn{2}{|c|}{ Hyperbolic method } & \multicolumn{2}{c|}{ Three-point method } & \multicolumn{2}{|c|}{$\begin{array}{c}\text { Asaoka } \\
\text { method }\end{array}$} & \multicolumn{2}{c}{$\begin{array}{c}\text { Combined } \\
\text { Forecasting model }\end{array}$} \\
\cline { 3 - 10 } & $\begin{array}{c}\text { Predicted } \\
\text { values } \\
(\mathrm{mm})\end{array}$ & $\begin{array}{c}\text { Relative } \\
\text { error } \\
(\%)\end{array}$ & $\begin{array}{c}\text { Predicted } \\
\text { values } \\
(\mathrm{mm})\end{array}$ & $\begin{array}{c}\text { Relative } \\
\text { error } \\
(\%)\end{array}$ & $\begin{array}{c}\text { Predicted } \\
\text { values } \\
(\mathrm{mm})\end{array}$ & $\begin{array}{c}\text { Relative } \\
\text { error } \\
(\%)\end{array}$ & $\begin{array}{c}\text { Predicted } \\
\text { values } \\
(\mathrm{mm})\end{array}$ & $\begin{array}{c}\text { Relative } \\
\text { error } \\
(\%)\end{array}$ \\
\hline 1 & 0.57 & 0.9015 & 0.5816 & 0.7890 & -0.2120 & 1.3110 & 1.3000 & 0.6532 & 0.1459 \\
\hline 2 & 2.45 & 2.3540 & -0.0392 & 2.4436 & -0.0805 & 2.7327 & 0.1154 & 2.5146 & 0.0264 \\
\hline 3 & 4.30 & 3.7232 & -0.1341 & 4.0418 & -0.1040 & 4.0722 & -0.0530 & 4.3463 & 0.0108 \\
\hline 4 & 4.62 & 5.0091 & 0.0842 & 4.8428 & 0.1410 & 5.3296 & 0.1536 & 4.6631 & 0.0093 \\
\hline 5 & 6.36 & 6.2118 & -0.0233 & 6.3034 & 0.0267 & 6.5047 & 0.0228 & 6.3859 & 0.0041 \\
\hline 6 & 7.32 & 7.3312 & 0.0015 & 7.3372 & 0.0445 & 7.5976 & 0.0379 & 7.3363 & 0.0022 \\
\hline 7 & 8.66 & 8.3673 & -0.0338 & 8.5169 & -0.0028 & 8.6084 & -0.0060 & 8.6631 & 0.0004 \\
\hline 8 & 8.85 & 9.3202 & 0.0531 & 9.0872 & 0.0750 & 9.5369 & 0.0776 & 8.8512 & 0.0001 \\
\hline 18 & 14.40 & 14.2691 & -0.0091 & 14.2299 & -0.0051 & 14.3020 & -0.0068 & 14.3995 & -0.0001 \\
\hline 19 & 14.25 & 14.3060 & 0.0039 & 14.2483 & 0.0218 & 14.3265 & 0.0054 & 14.1902 & -0.0042 \\
\hline 20 & 14.53 & 14.2597 & -0.0186 & 13.8251 & 0.0164 & 14.2688 & -0.0180 & 14.4628 & -0.0046 \\
\hline 21 & 14.71 & 14.1300 & -0.0394 & 13.8603 & 0.0165 & 14.1288 & -0.0395 & 14.7229 & 0.0009 \\
\hline
\end{tabular}

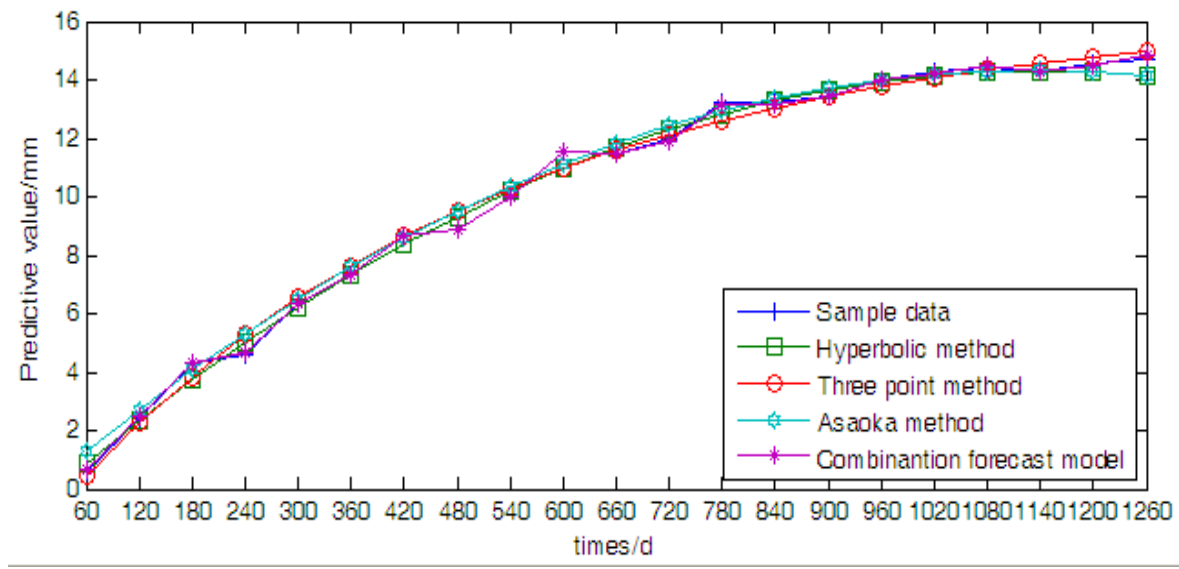

Figure 2. The Predictive Effect of four Kinds of Forecast Methods

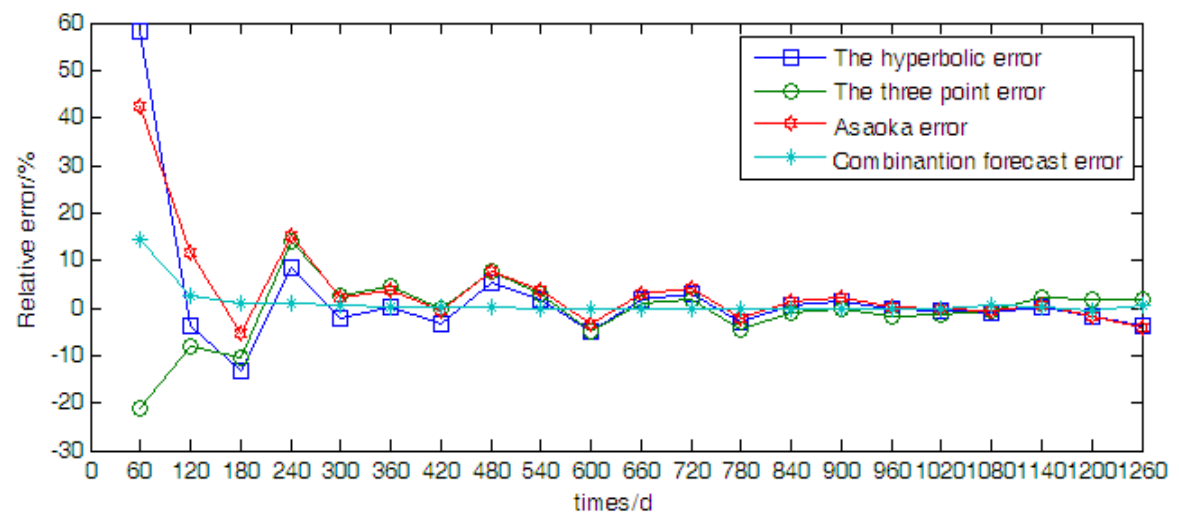

Figure 3. The Relative Error of Four Kinds of Forecast Methods 
From Figure 2 and Figure 3, we can see that the subgrade settlement data of Hyperbolic and Asaoka methods are volatile in the early, while the forecast error were largely, mainly due to the methods defined in the model themselves are relatively simple. The Relative errors have opposite direction between three-point method and the first two methods. Considering the characteristics and the forecast results of single forecast method, Combination Forecast model improve the forecast accuracy, using LSSVM to assign different rights to the single forecast methods.

4.3.1. The impact of the forecast starting time on the forecasting results: Training on the forecast model by using sample data with different predict staring time, obtaining the Combination Forecast model's accuracy assessment value, respectively. From Table 3 we can see, that the range of subgrade settlement change largely in the previous test, and the process of settlement is unstable, in order to improve the forecast accuracy of the model, we can delay forecast starting time appropriately.

\section{Table 3. The Impact of the Forecast Starting Time on the Forecasting} Results

\begin{tabular}{c|c|c|c}
\hline starting time(day) & RMSE & CC & MAPE (\%) \\
\hline 60 & 0.0042 & 0.9960 & 0.0163 \\
\hline 120 & 0.0026 & 0.9976 & 0.0144 \\
\hline 180 & 0.0022 & 0.9987 & 0.0099 \\
\hline
\end{tabular}

4.3.2. The impact of interval time on the forecasting results: Training on the forecast model by using the sample data with different time interval, obtaining the Combination Forecast model's accuracy assessment value, respectively. From Table 4 we can see, that the forecast interval is shorter, the higher the forecast accuracy.

Table 4. The Impact of Interval Time on the Forecasting Results

\begin{tabular}{c|c|c|c}
\hline Interval time(day) & RMSE & CC & MAPE (\%) \\
\hline 35 & 0.0034 & 0.9976 & 0.0139 \\
\hline 60 & 0.0042 & 0.9963 & 0.0144 \\
\hline 92 & 0.0051 & 0.9868 & 0.0206 \\
\hline
\end{tabular}

The above analysis can be seen, the forecast starting time and interval time have a certain effect on the forecast result of the Combination Forecast, but the overall forecast accuracy of Combination Forecast model still meet the needs of practical engineering. In the application of practical, we need to fully consider the effect of the early fluctuations of subgrade settlement, select the appropriate starting time for training forecast model; while predictive time interval should not be too long, should be about 30 days is appropriate.

\section{Conclusion}

Based on the characteristics of the subgrade settlement single forecast model, the Combination Forecast model is constructed. The forecasting effects of Combination 
Forecast model are verified by testing the actual engineering data, the following conclusions are obtained:

(1) When selecting the single forecast model, based on the forecast accuracy, the single forecast model with opposite relative error deviation direction and approximated error magnitude are selected as the inputs of Combination Forecast model.

(2) The least squares support vector machine is adopted as an output. Its forecasting process is regardless of time, but is related to the forecasting values of the single forecast model. So a number of single forecast models with small relative error and peer deviate direction are selected, which will effectively improve the forecasting results of Combination Forecast model.

\section{Acknowledgements}

This work was supported by Applied Fundamental Research Plan Project of Sichuan Province of China (No. 2013JY0179), and Science and Technology Supporting Plan Project of Sichuan Province of China (No. 2013GZX0167).

\section{References}

[1] M. B. Jain, M. K. Nigam and P. C. Tiwari, "Curve fitting and regression line method based seasonal short term load forecasting", Information and Communication Technologies (WICT), (2012); Trivandrum, India.

[2] L. Jiaguo and L. Fei, "Generalized Hyperbolic-function Method with Computerized Symbolic Computation to the Nizhnik-Novikov-Veselov Equation", Computer Distributed Control and Intelligent Environmental Monitoring (CDCIEM), (2012); Hunan, China.

[3] G. Yang, R. Rao, K. Wu, Y. Li and X. N. Bao "Prediction of compressive strains at the top of subgrade based on BP neural network Remote Sensing", Environment and Transportation Engineering (RSETE), (2011); Nanjing, China.

[4] Y. Hong, L. Fei and X. Yuge, "Robust control based on LS-SVM for uncertain nonlinear system", Cybernetics and Intelligent Systems, (2008); Chengdu, China.

[5] H. Erdem, "Prediction of the moment capacity of reinforced concrete slabs in fire using artificial neural network", Advances in Engineering Software, Elsevier Ltd Publisher, vol. 41, no. 2, pp. 270-276.

[6] Z. Hui, L. Bin and Z. Zhuo-qun, "A short-term wind speed forecasting simulation research based on ARIMALSSVM combination method", Materials for Renewable Energy \& Environment (ICMREE), (2011); Shanghai, China.

[7] F. Shengyang, "Settlement prediction of high-speed railway subgrade based on least squares support vector machine", Zhongguo Tiedao Kexue/China Railway Science, vol. 33, no. 6, (2012), pp. 6-10.

[8] J. P. Guo and W. H. Li, "Regression Analysis of Interval Data Based on Error Theory", Networking, Sensing and Control, (2008); Sanya, China. 